\title{
Bienestar y resultados zootécnicos en conejas de aptitud carne. Revisión bibliográfica
}

\author{
M. López ${ }^{1, *}$, C. Cervera ${ }^{2}$ y J.J. Pascual ${ }^{2}$
}

1 Dpto. Producción Animal y Ciencia de los Alimentos, Facultad de Veterinaria, Universidad de Zaragoza, Miguel Servet 177. 50013 Zaragoza, España

2 Instituto de Ciencia y Tecnología Animal, Universitat Politècnica de València, Camino de Vera s/n. 46022 Valencia, España

\section{Resumen}

El manejo de reproductoras en grupo o semigrupo, con objeto de mejorar su bienestar, proporciona solo un ligero enriquecimiento del comportamiento locomotor y social, y algunos resultados zootécnicos próximos pero estadísticamente inferiores a los obtenidos en jaula individual, sin conseguir así compensar los resultados negativos del método: mayor mortalidad de gazapos lactantes, estrés, agresividad, heridas y mortalidad de las madres, unido a la complejidad, tiempo y trabajo requeridos por este manejo. Enriquecer la jaula individual mediante plataforma no deteriora los resultados zootécnicos, permite incrementar la movilidad y el repertorio comportamental de las conejas (saltar, erguirse), aumentando además la superficie disponible, pero empeora la higiene y aumentan los problemas sanitarios (pododermatitis, fracturas). Reposapatas y elementos para roer, así como refinar el enriquecimiento en las jaulas estándar, parece una vía útil para mejorar el bienestar mientras se profundiza en la gestión de la respuesta agresiva, así como en la idoneidad y en el modelo de grupo en las reproductoras de carne.

Palabras clave: Conejas reproductoras, enriquecimiento jaula individual, manejo en grupo, comportamiento, agresividad, salud.

\section{Welfare and performance traits of meat rabbit females. A review}

\begin{abstract}
Continuous and part-time group housing systems have been tested to improve the welfare of reproducing rabbit does. Part-time group provides some improvements in locomotor and social behavior, some good reproductive results -still significantly lower than individual cages-, without making up for its negative effects: higher kit mortality, rates of aggression, injured and does mortality, on top of the complexity, time and effort invested. The continuous group system increases more the aforementioned problems. Individual cages enriched by a platform have no negative effect on productive performances, provide more opportunities for movements and behavioral repertoire in does (jumping, stand up), increase space availability, but deteriorate hygiene and health (pododermatitis, fractures). Plastic footrests, gnawing blocks, have a positive impact on improving welfare in rabbits. In conclusion, refining the enrichment of standard cages seems a useful way to improve welfare while studying how to reduce aggression, as well as the suitability of the breeding group and the group model in the meat rabbit does.
\end{abstract}

Keywords: Rabbit does, enrichment individual cages, group-housing, behavior, aggression and injuries, health.

* Autor para correspondencia: marina@unizar.es

Cita del artículo: López M, Cervera C, Pascual JJ (2020). Bienestar y resultados zootécnicos en conejas de aptitud carne. Revisión bibliográfica. ITEA-Información Técnica Económica Agraria 116(2): 131-149. https://doi.org/10.12706/itea.2020.002 


\section{Introducción}

El bienestar animal es un factor más de la producción ganadera, como la alimentación, la genética, o la reproducción. Al igual que en estas otras disciplinas, el bienestar se ha procurado de manera intuitiva, empírica y en base al sentido común en sus inicios, y ahora empieza a ser aplicado de forma reglada, deseablemente en función de los resultados de los trabajos técnico-científicos. Pero para establecer reglas es necesario encontrar respuestas a las preguntas y a los problemas que se plantean, $y$, como ambos son numerosos y las respuestas frecuentemente urgentes, nos encontramos en la vorágine de prestar atención a los acontecimientos y al cuestionamiento generalizado. Esto ocurre especialmente en la especie cunícola, para la que "intrínsecamente se relaciona el estar en una jaula con falta de bienestar", como han señalado algunos expertos. En efecto, las jaulas individuales de alambre galvanizado constituyen el alojamiento habitual de la coneja y su camada hasta el destete, tanto en la producción de carne en granjas intensivas como en la mayoría de los núcleos de autoconsumo o en cunicultura alternativa (González-Redondo et al., 2015). Aunque no se han encontrado indicadores de graves problemas de bienestar de los animales, este modelo de producción ha sido cuestionado porque la estancia en la jaula individual limita los comportamientos de locomoción y de contacto social reconocidos en los conejos silvestres, y habitualmente se hace la comparación directa entre ambos grupos, como si la adaptación y la habituación de los animales a su entorno consecuentes a la domesticación no hubieran existido, lo cual es erróneo (Carneiro et al., 2014). Esta sistemática comparación se debe a que los conejos domésticos mantienen comportamientos similares a los silvestres, por ejemplo, en las relaciones materno-filiales (lactación de alrededor de cinco minutos cada 24 horas, escasa atención al nido aparentemente, ...), reproducción (elevada tasa de aceptación al macho inmediatamente después del parto, ...), o alimentación (práctica de la cecotrofia, ...). Como expondremos en la presente revisión, también sus relaciones sociales son comparables, caracterizándose las conejas silvestres por el establecimiento de un rígido orden de jerarquías (separadamente del de los machos), presentando las subordinadas alto nivel de estrés, menor éxito reproductivo y reducción de la vida reproductiva respecto a las dominantes (von Holst et al., 1999; DiVincenti y Rehrig, 2016). Estas similitudes no dejan de ser lógicas ya que se trata de una única especie.

A estas características peculiares de producción en la especie cunícola, se añade la singular gestión político/social del bienestar animal, aspecto también especialmente interesante, pues mientras que hay relativamente poca legislación a aplicar en granja en relación con la ciencia zootécnica bien establecida -genética, reproducción o alimentación-, en relación con bienestar se pretenden aplicar normativas en muchos casos con escaso fundamento científico, ocasionando problemas innecesarios que acaban repercutiendo de forma negativa sobre la salud y el propio bienestar de las reproductoras. En esta especie, la salud, o mejor, la mortalidad ha sido reconocida como el primer indicador de bienestar en los conejos (Hoy y Verga, 2006). Los otros indicadores son, por este orden: morbilidad (patologías; heridas); fisiología (niveles hormonales; tasa cardiaca; respuestas inmunológicas); comportamiento (etograma; test de comportamiento); y resultados productivos (crecimiento; conversión alimenticia; tasa de fertilidad).

En el presente trabajo se revisan los resultados de experimentos destinados a mejorar las condiciones de bienestar de las conejas reproductoras de aptitud carne. Las innovaciones que se estudian sobre estas conejas con 
vistas a su implementación en la cunicultura intensiva hacen referencia al manejo de las conejas en grupo, usualmente de 4 a 8 conejas, como contraposición al manejo individual mencionado (aspecto relativo a la mejora del comportamiento social), al aumento del tamaño de las jaulas estándar (aspecto relacionado con la mejora del comportamiento locomotor y con la disponibilidad de espacio) y, por último, al enriquecimiento de las jaulas estándar (con fines de entretenimiento y desarrollo de comportamientos específicos). También se mencionan algunas condiciones de manejo relacionadas con la actividad reproductiva de las conejas (duración del ciclo productivo, uso de feromonas, vía de utilización de la hormona GnRH) que podrían influir sobre el bienestar de las mismas. Los objetivos de los experimentos que se presentan se dirigen a la valoración del enriquecimiento etológico que dichas innovaciones aportan así como a la evaluación del nivel de estrés, el control de los resultados productivos y de la viabilidad de los animales.

\section{Peculiaridades de la especie cunícola en relación con el bienestar animal}

Además de su presencia en la naturaleza en estado silvestre, otra faceta que diferencia a esta especie de la mayoría de las otras de interés zootécnico en relación con el bienestar animal es su cada vez más extendida presencia como animal de compañía. En esta aptitud, el carácter social de los conejos está ampliamente considerado, indicando Mullan y Saunders (2019) que su motivación para la compañía de otros conejos es casi tan alta como la del alimento. Para facilitar este comportamiento sin problemas de agresividad, la Rabbit Welfare Association \& Fund (RWAF) (RWAF, 2019) indica que "Castrating male rabbits and spaying females is vital for many reasons, one of which is that it will help reduce aggressive behaviour, particularly towards each other". Similar procedimiento sugiere el Gobierno Escocés en su reciente guía para el bienestar de los conejos de compañía (Scottish Government, 2018), así como los expertos en pet rabbits antes mencionados, Mullan y Saunders (2019). Como ventajas que se indican en estos textos, se destaca que en machos la castración facilita la vida social, reduce las peleas con otros machos, aminora los sprays de orina y el comportamiento sexual con los humanos; en hembras también facilita la vida social, previene carcinomas uterinos, impide la preñez si están con machos enteros, y puede reducir las agresiones a los dueños.

En su faceta como animal de laboratorio, a pesar de muchos intentos de alojamiento en grupo, el éxito ha sido pequeño, relegando a estos conejos a espacios individuales en los centros de investigación según indican DiVincenti y Rehrig (2016). También se ha propuesto la castración como procedimiento para evitar la agresividad cuando se forman grupos, pero ya hace años se señaló que esta práctica podría modificar la fisiología de los conejos $\mathrm{y}$, consecuentemente, los resultados de las pruebas experimentales (BVAAWF / FRAME / RSPCA /UFAW, 1993), planteando estos autores si el uso de un sistema de alojamiento determinado justifica una intervención quirúrgica. No conocemos estudios científicos que comparen la castración con otros procedimientos en relación con el enriquecimiento de la vida social de estos conejos, pero tal estado sin duda reduce los comportamientos naturales que la sociedad quiere potenciar en los animales de producción. En la presente revisión solo se hará referencia a estos últimos, concretamente a los destinados a la producción de carne, como previamente se ha indicado. 


\section{Manejo de las reproductoras en grupo}

En relación con el manejo en grupo, una opinión extendida en la sociedad occidental es creer apropiado que los conejos domésticos vivan y críen en colonias, imitando el vivar comunal de los conejos silvestres. Las cuestiones a resolver aplicando este manejo serían, en primer lugar, comprobar si se enriquece el patrón de comportamientos cuando varias conejas domésticas se reproducen en una misma jaula, y, en segundo y más importante, si efectivamente mejora su bienestar respecto a la jaula individual. Es necesario considerar ambos aspectos pues, aunque referido a los gazapos de cebo, hace años que Mirabito et al. (2000) indicaban que "desde el punto de vista etológico algunos autores piensan que poner madera o alfalfa a disposición de los gazapos es un manejo adecuado. Nuestros resultados muestran que desde el punto de vista del bienestar esto no es así, porque hay mayores niveles de mortalidad o mayor riesgo".

De acuerdo con lo anterior, los diseños experimentales han evolucionado empujados por las respuestas comportamentales de las conejas, especialmente por las respuestas negativas, las cuales están claramente ligadas a la agresividad propia del comportamiento jerárquico de las conejas silvestres y domésticas siempre que dos o más adultos comparten espacio. Así, se ha observado que las reproductoras alojadas en grupo pelean entre sí, se provocan heridas y sufren un importante estrés crónico, pues presentan concentraciones tan elevadas como $175 \mathrm{nmol} / \mathrm{g}$ de corticosterona fecal en grupos constituidos por 4 hembras y 1 macho (monta en libertad), respecto a los 54 y $61 \mathrm{nmol} / \mathrm{g}$ mostrados por conejas en jaula individual manejadas en ciclo de 42 (inseminación día 11 post-parto) y 33 días (inseminación día 2 post-parto), respectivamente (Szendrö et al., 2013). La reproducción en grupo también aumenta la frecuencia de pseudo-gestaciones ( $23 \mathrm{vs.} 0 \%$ en jaula individual), en gran parte como con- secuencia del comportamiento de monta de las otras conejas del grupo (Rommers et al., 2006). Además crece la mortalidad de los gazapos, multiplicándose globalmente por dos respecto a las jaulas individuales según resultados de Mirabito et al. (2005a), pudiendo ser todavía más elevada (38,5 vs. $15,2 \%)$ (Szendrö et al., 2013). Esta alta mortalidad se atribuye a la frecuente concurrencia de dos o incluso tres partos en el mismo nidal (Mirabito et al., 2005a), así como a la libre entrada de las conejas al nidal de las otras (Ruis y Coenen, 2004), y a que los gazapos mayores invaden nidos ajenos ocupados por camadas más jóvenes (Ruis et al., 2005), acumulándose más de 20 gazapos en algunos nidales mientras otros quedan vacíos (Machado et al., 2016). Como factores responsables de la baja tasa de supervivencia también se han señalado la competencia por la leche de las madres (Cervera et al., 2016), la competencia por los nidos (Mirabito et al., 2005a; Szendrö et al., 2013), así como el comportamiento agresivo, pues, en ocasiones, los gazapos se encuentran en el suelo fuera de los nidales debido a que los adultos los echan de los mismos (Szendrö et al., 2013), o hay episodios de ataques de las hembras a los gazapos ajenos, particularmente cuando han nacido en distinto momento (Ruis, 2006).

Previamente al inicio de la actividad reproductiva, en experimentos con grupos de cuatro conejas nulíparas se ha observado que, si tienen posibilidades de elegir, su preferencia por permanecer solas en su propia jaula o con una única compañera es más elevada que su estancia en los espacios comunes (Matics et al., 2017). A su vez, trabajando con conejas de recría entre 12 y 18 semanas de vida, Martínez-Paredes et al. (2019) han encontrado que el alojamiento en grupos grandes (16 a 24 individuos) penaliza el crecimiento y aumentan las lesiones presentes en el momento de la inseminación respecto a los grupos reducidos (2 a 4 animales), y el efecto es mayor cuanto mayor es la densidad. 
En función de los resultados mencionados, el diseño de las experiencias para el estudio de las reproductoras en grupo ha evolucionado notablemente. Así, desde los trabajos iniciales caracterizados por el uso de recintos con cama de paja y grupos constituidos por dos, tres o cuatro conejas y presencia continua de un macho para conseguir monta natural espontánea (Hoy y Schuh, 2004; Ruis y Coenen, 2004; Szendrö et al., 2010), que normalmente acontece en el post-parto (Ruis, 2006; Szendrö et al., 2013), se ha pasado en la actualidad a la utilización de inseminación artificial, manejo en ciclos de 42 días mayoritariamente y posibilidades de identificación individual electrónica para que cada hembra pueda acceder a un único nidal (Ruis, 2006; Rommers et al., 2012). Asimismo, se ha cambiado de mantener a las reproductoras continuadamente en grupo al estudio del manejo en grupo temporal, es decir, las conejas comparten jaula en una fase del ciclo y en la otra fase cada coneja ocupa una jaula individual. Este sistema se conoce como "semigrupo" o "part-time". Su objetivo es evitar las peleas ligadas al parto y a la protección de la reciente prole, de modo que las conejas están separadas desde 3 días antes del parto hasta que los gazapos empiezan a salir del nido, lo que acontece hacia los 18 días de edad (Andrist et al., 2012; Machado et al., 2016; Cervera et al., 2017). De este modo, las reproductoras están en sus jaulas individuales durante 21 días y en la jaula colectiva los otros 21 días del ciclo, si bien en los trabajos más recientes se acorta el tiempo de estancia en grupo porque se retrasa el momento del reagrupamiento de las conejas al 21 o 23 días de edad de los gazapos (Maertens y De Bie, 2017; Rommers y de Greef, 2017). La razón para este retraso es que con 18 días algunos gazapos de pequeño tamaño no encuentran el bebedero, además de permanecer mucho tiempo en el nidal y tener, consecuentemente, menor probabilidad de amamantarse durante el alojamiento en grupo (Maertens y de
Bie, 2017). Además, los gazapos de 18 días de edad son inmaduros y su ingesta de sólidos es escasa, incluso 21 días no parece todavía edad idónea para constituir el grupo, mientras que con 23 días ya se estiman suficientemente maduros (Rommers y de Greef, 2017). Para conseguir este agrupamiento temporal, se favorece o se impide el contacto entre las hembras mediante paredes móviles en las jaulas, o mediante puertas que puedan cerrarse o abrirse, permitiendo la formación del grupo o la individualización de las reproductoras a juicio del cuidador. No obstante, sea cual sea el protocolo, la agresividad y las lesiones consecuentes al restablecimiento de la jerarquía (Buijs et al., 2015) así como el estrés, medido en función de la evolución de la temperatura corporal (Graf et al., 2011; Andrist et al., 2014) (Figura 1) y del nivel de glucocorticoides (Szendrö et al., 2016; Ródenas et al., 2019), se reactivan cada vez que las hembras vuelven a reunirse. Estas manifestaciones negativas son especialmente frecuentes en las conejas más jóvenes (Ródenas et al., 2019) y también cuando los grupos no son estables porque se incorpora reposición (Andrist et al., 2012). Además, agresividad, lesiones y estrés se observan incluso cuando se enmascaran los aromas naturales del grupo mediante determinadas sustancias como vinagre o alcohol (Andrist et al., 2014).

El diseño de la jaula individual y de su transformación a colectiva parece que podría condicionar la respuesta agresiva de las conejas según Rommers y de Greef (2017). Estos autores realizan varias pruebas modificando de manera sencilla el diseño de su prototipo de jaula (colocación de paneles de madera sobre o bajo la plataforma, en dirección transversal u horizontal, eliminación de la paredes laterales de los nidales...) y encuentran que, en sus condiciones, el número de conejas heridas se reduce cuando se retiran las paredes de los nidales, es decir, cuando la jaula colectiva pasa a tener un diseño comparable a un parque de cebo. 




Figura 1. Evolución de la temperatura corporal, determinada mediante monitorización, en conejas manejadas en grupo que se reagrupan en el parque que habían compartido antes de la separación (familiar) o se reagrupan en un parque diferente, limpio y desinfectado (nuevo). BR (antes del reagrupamiento); $90 \mathrm{~min}$, 1d, 3d, 6d (90 minutos, 1 día, 3 días, 6 días tras el reagrupamiento). • Significativamente distinto $(P<0,05)$. Figura obtenida de Graf et al. (2011).

Figure 1. Evolution of body temperature monitored in rabbit females managed in group housing, that are regrouped using the pen they had shared before the separation (familiar) or are regrouped in a different clean and disinfected pen (nuevo). Before (BR) and $90 \mathrm{~min}, 1 \mathrm{~d}, 3 \mathrm{~d}$ and $6 \mathrm{~d}$ after regrouping. $\bullet$ Significant differences $(P<0.05)$. Figure obtained from Graf et al. (2011). 
Criterios productivos importantes como la prolificidad o el crecimiento de la camada lactante obtenidos en algunos de los trabajos de cría en grupo o semi-grupo no difieren de los presentados por conejas alojadas continuamente en jaula individual (Mirabito et al., 2005a; Ruis, 2006). Por el contrario, se ha observado una importante reducción de la fertilidad tanto en el experimento de Mirabito et al. (2005a), estudiando grupos de 4 conejas con inseminación artificial y ciclo de 42 días (51 vs. $80 \%$ ), como en el de Rommers et al. (2006), con grupos de 8 conejas y similar manejo reproductivo $(55,6$ vs. $84,2 \%$ ) (Tabla 1). Szendrö et al. (2013) comparan los resultados de grupos formados por 4 hembras y un macho con monta natural respecto a los de conejas que ocupan una jaula individual y se inseminan a los 2 o a los 11 días post parto, encontrando tasas de fertilidad muy bajas en el primer tratamiento $(45,6 \%)$, respecto a los otros $(77,6$ y $85,2 \%$, respectivamente). La baja fertilidad se explica en parte por las pseudo-gestaciones antes mencionadas ( $23 \%$ en grupo vs. $0 \%$ en jaula individual) (Rommers et al., 2006). También se ha observado un menor número de gazapos vivos al nacimiento (Semigrupo: Machado et al., 2016) o inferior tamaño y/o peso de la camada al destete (Grupo: Ruis et al., 2005; Rommers et al., 2006; Semigrupo: Cervera et al., 2017), tal vez porque las hembras en grupo muestran amamantamientos cortos y con intervalos de más de $24 \mathrm{~h}$ entre ellos, según constatan Rommers et al. (2012). Como excepción, en el experimento en semigrupo de Machado et al. (2016) se han hallado pesos al destete más elevados en los gazapos

Tabla 1. Resultados reproductivos y evolución de la camada hasta el destete en conejas alojadas en jaula individual vs. conejas mantenidas en grupo continuo constituido por ocho hembras. Reproducción mediante inseminación artificial día 11-post-parto, ciclo de 42 días. Tabla obtenida de Rommers et al. (2006).

Table 1. Reproductive performance of rabbit females and evolution of their litter until weaning when housed in an individual cage vs. a continuous group of eight females. Reproduction by Al at 11 days postpartum, with a cycle of 42 days. Table obtained from Rommers et al. (2006).

\begin{tabular}{lccc}
\hline & \multicolumn{2}{c}{ Tratamiento } & $P$ \\
\cline { 2 - 3 } & Individual & Grupo & \\
\hline Camadas (n) & 101 & 102 & \\
Fertilidad (\%) & 84,2 & 55,6 & 0,001 \\
Gazapos: & & & \\
Nacidos total (n) & $8,9 \pm 0,3$ & $8,4 \pm 0,3$ & 0,19 \\
Nacidos vivos (n) & $8,4 \pm 0,3$ & $7,7 \pm 0,3$ & 0,16 \\
Mortalidad 14 d (\%) & 7,4 & 10,1 & 0,15 \\
Peso 14 d (g) & $241 \pm 11$ & $233 \pm 12$ & 0,60 \\
Destetados (n) & $6,9 \pm 0,2$ & $6,6 \pm 0,5$ & 0,58 \\
Peso destete (g) & $841 \pm 12$ & $720 \pm 25$ & 0,001 \\
\hline
\end{tabular}

$P$ : significación estadística $(P<0,05)$. 
nacidos en jaulas colectivas. Los autores atribuyen este resultado a una mayor ingesta, tanto por parte de los gazapos como de sus madres, y a pérdidas de reservas más acusadas en el alojamiento en semigrupo que las de conejas alojadas continuamente en jaula individual.

Otros resultados que cuestionan el sistema de manejo en grupo o semigrupo serían el acortamiento de la vida productiva que encuentran Ródenas et al. (2019), así como el mayor número de reproductoras eliminadas o muertas, llegando solo 4 reproductoras al final del experimento entre las alojadas en grupo $(21 \%)$ respecto a 8 en las situadas en jaula individual (42\%) en el trabajo de Cervera et al. (2017) (Tabla 2). También Szendrö et al. (2013) encuentran tasas de supervivencia de $50 \%$ en grupo vs. $71-81 \%$ en jaula individual. La elevada mortalidad de gazapos lactantes $(17,6 \%$ en parque colectivo de cuatro conejas vs. $10,6 \%$ en jaulas con plataforma con dos reproductoras vs. $8,7 \%$ en jaula individual estándar; Mirabito et al., 2005a), el riesgo de coccidiosis cuando en el suelo de la jaula colectiva se usa cama de paja, y la presencia de pododermatitis aún con suelos de rejilla plástica, posiblemente por falta de higiene (Ruis, 2006), son también resultados que limitan el sistema. En algún experimento se suman la mayoría de los problemas. Por ejemplo, Szendrö et al. (2010) encuentran que más de la mitad de las conejas del grupo no pare, por lo que la fertilidad es baja, la mortalidad en nido es elevada y el número de destetados $/ \mathrm{m}^{2}$ inferior al $20 \%$ de los obtenidos en jaula individual; además de que la supervivencia es escasa en las conejas por la alta tasa de sacrificios consecuentes a heridas $50 \%$ de las conejas del grupo) y sus niveles de corticoides en heces muy elevados, tal como antes hemos indicado (Szendrö et al., 2013).

Por todas estas razones, Mirabito et al. (2005a, 2005b), Szendrö y McNitt (2012) y Matics et al. (2017), entre otros autores, no recomien- dan el sistema de cría en grupo o semigrupo ni desde la óptica del bienestar, ni de los resultados productivos y sanitarios, indicando que, si bien vivir en colonias es decisivo para la supervivencia en los conejos silvestres, para los conejos domésticos solo debería proponerse el alojamiento individual (Szendrö, 2009). Alguna experiencia ha tenido éxito desde el punto de vista productivo según indican sus autores, pues con manejo de 21 días en grupo y 21 días en jaula individual, inseminación artificial y sistema "todo dentro todo fuera" han conseguido resultados generales muy buenos y bastante cercanos, aunque estadísticamente inferiores, a los de las jaulas individuales (fertilidad entre 83 y $90 \%, 12$ gazapos nacidos vivos, 10 gazapos destetados/parto; Maertens y Buijs, 2016). Estos autores también observan una mejora del comportamiento locomotor y de las interacciones sociales, pero concluyen que dicha mejora es pequeña, no encontrando una clara evidencia de que los cambios conductuales que generan compensen, en términos de bienestar animal, el alto porcentaje de conejas heridas (Buijs et al., 2015; Maertens et al., 2015), o la mortalidad de los gazapos lactantes (Maertens y De Bie, 2017) consecuentes al reagrupamiento temporal. Estos resultados y conclusiones son similares a los mencionados en los trabajos iniciales de mejora del bienestar mediante cría colectiva en grupos de cuatro conejas (Mirabito et al., 2005b), y confirman también los problemas de mortalidad, peleas y aptitudes defensivas, sobre todo en el periodo de partos, descritos a principios de los años 60 (Paillard, 1970) y 70 (Lecerf, 1982, cit. Mirabito et al., 2005a) en el sistema de producción "en colonia", inconvenientes que condujeron a la desaparición del sistema (Mirabito et al, 2005a).

Szendrö et al. (2016) indican que para los conejos silvestres la vida en grupo tiene una relación beneficio/coste muy positiva, porque los costes correspondientes a la agresividad 
Tabla 2. Resultados reproductivos y evolución de la camada hasta el destete en conejas alojadas en jaula individual vs. conejas mantenidas en semi-grupo de seis hembras. Reproducción mediante inseminación artificial día 11 post-parto, ciclo de 42 días (21 días en jaula individual, desde 3 preparto hasta 18 postparto, y 21 días en grupo). Tabla obtenida de Cervera et al. (2017).

Tabla 2. Reproductive performance of rabbit females and the evolution of the litters until weaning when housed in an individual cage vs. a semi-group of six females. Reproduction by Al 11 days post-partum, with a cycle of 42 days (21 days in individual cage, from 3 pre-partum to 18 post-partum, and 21 days in group). Table obtained from Cervera et al. (2017).

\begin{tabular}{lcccc}
\hline & Individual & Semi-grupo & ES & P \\
\hline Conejas (n): & 19 & 19 & & \\
Vida (días) & 180 & 152 & 22,0 & 0,3804 \\
Finalizan 5 partos & 8 & 4 & & \\
Partos (n): & 58 & 44 & & \\
Inseminaciones & 1,5 & 1,6 & 0,09 & 0,5265 \\
Nacidos totales & 9,9 & 10,5 & 0,7 & 0,4345 \\
Nacidos vivos & 8,9 & 9,5 & 0,6 & 0,5071 \\
Peso gazapo vivo (g) & 58 & 56 & 2,05 & 0,2976 \\
Lactación coneja: & & & & \\
Peso (g) & 4514 & 4416 & 40 & 0,0919 \\
Consumo (g MS) & 344 & 317 & 4,8 & 0,0001 \\
Grosor depósitos grasos (mm) & 7,0 & 6,7 & 0,08 & 0,0390 \\
Camadas: & 10,1 & & & \\
Tamaño a 18 días & 10,0 & 9,4 & 0,15 & 0,0100 \\
Tamaño destete & 9,8 & 9,2 & 0,15 & 0,0100 \\
Mortalidad parto a 18 días (\%) & 0,6 & 14,3 & & 0,0814 \\
Mortalidad 18 a 28 días (\%) & 264 & 253 & 6,8 & 0,0214 \\
Peso gazapo a 18 días (g) & 531 & 492 & 6,8 & 0,0204 \\
Peso gazapo a destete (g) & & & & \\
\hline
\end{tabular}

ES: error estándar. $P$ : significación estadística $(P<0,05)$. MS: materia seca.

resultante de la competitividad (por comida, apareamiento, nidales...) y a la existencia de individuos dominados (más estresados, con menos fertilidad, prolificidad y viabilidad, menor esperanza de vida...), se acompañan de beneficios importantes, tales como el me- nor riesgo de predación, la cooperación en la construcción de la madriguera y la protección y supervivencia en la misma, rico comportamiento social, dominio de un territorio, etc. Sin embargo, en el caso de los conejos domésticos a costes "biológicos" similares para 
el animal (más un incremento en la complejidad o en el tiempo de trabajo por parte del cuidador, así como un aumento del coste de producción que acompaña al sistema de grupo) le corresponde únicamente un cierto aumento del comportamiento locomotor y de interacción social, lo cual se traduce en un escaso peso en esa relación beneficio/coste, por lo que su papel en la mejora de las condiciones reales de bienestar de los individuos y de la población es cuestionado.

\section{Enriquecimiento de la jaula maternal mediante aumento del espacio disponible}

Antes de revisar este apartado es importante indicar que en algunos experimentos las conejas no muestran preferencia por las jaulas de mayor tamaño y su elección parece estar relacionada con factores como el tamaño de la jaula en la que hicieron el parto (Mikó et al., 2012), aunque otros autores han constatado la elección de espacios más amplios mediante la aplicación de test específicos de preferencia y motivación (Cervera et al., 2018). Por otra parte, no está claro que las conejas modifiquen sus comportamientos o sus resultados productivos como consecuencia del aumento de la superficie (3420 vs. $5855 \mathrm{~cm}^{2}$ ) (Mirabito et al., 2005c), ni tampoco que precisen jaulas de más de $40 \mathrm{~cm}$ de altura para posicionarse sobre sus patas traseras, porque no le dan uso ni en el $1 \%$ de su tiempo, según observan Negretti et al. (2010).

A pesar de lo indicado, el incremento de la superficie de la jaula estándar se ha considerado otro procedimiento para mejorar el bienestar de las conejas. A nivel práctico se ha propuesto este aumento mediante la colocación en el interior de la jaula de una plataforma situada a $30-40 \mathrm{~cm}$ de altura sobre el suelo de la jaula. Hoy y Verga (2006) recomiendan unas dimensiones de $50 \mathrm{~cm}$ de anchura y $30 \mathrm{~cm}$ de profundidad para la plataforma. A título indica- tivo, en España la legislación para la protección de los conejos utilizados en experimentación y otros fines científicos, indica que estos animales deben contar con plataformas situadas a $25-30 \mathrm{~cm}$ de altura, que no deben cubrir más del $40 \%$ del suelo y que tendrán unas dimensiones desde $55 \times 25 \mathrm{~cm}$ hasta 60 $x 35 \mathrm{~cm}$, en función de la edad y del peso de los animales (Villalba, 2019).

Este elemento permite aumentar el repertorio de comportamientos específicos, como saltar o erguirse sobre sus patas posteriores, mientras que, paralelamente, se incrementa, la superficie real disponible. A nivel de granja la presencia de plataforma permite mayor movilidad, enriquece el repertorio comportamental de las conejas (Alfonso-Carrillo et al., 2014a) y parece reducir las agresiones consecuentes al reagrupamiento de reproductoras en el sistema de semigrupo (Rommers et al., 2014a). La plataforma es más utilizada por la coneja si es de material plástico respecto al alambre (Mikó et al., 2012), acortando el tiempo de utilización tanto del reposapatas como del suelo de rejilla de la jaula (Figura 2). Además, la plataforma puede permitir a la coneja separarse de su camada cuando los gazapos empiezan a salir del nidal y molestan a la madre porque se acercan a mamar (Mirabito, 2003; Alfonso-Carrillo et al., 2014a) (Figura 3), objetivo insuficientemente conseguido en algunos experimentos pues los gazapos suben a la plataforma ya con 17 días de edad, y cada vez la ocupan con más frecuencia hasta el destete (Mikó et al., 2012), sobre todo si es de plástico como se ha indicado. Por otra parte, jaulas con plataforma se han utilizado para valorar el interés de colocar comederos específicos para gazapos desde los 14 días de edad, diferenciados de otros comederos situados a $32 \mathrm{~cm}$ del suelo y sólo accesibles para la coneja, con repercusión positiva sobre el crecimiento de los gazapos lactantes $y$, por tanto, en su bienestar (Mirabito y Boquier, 2005). 




Figura 2. Localización durante $24 \mathrm{~h}$ de conejas lactantes o gestantes alojadas en jaulas estándar vs. jaulas con plataforma, ambas provistas de reposapatas. ${ }^{a}$, b, c Valores para el suelo enrejillado no compartiendo superíndice difieren significativamente $(P<0,05)$. ${ }^{A}, B$ Valores para el reposapatas no compartiendo superíndice difieren significativamente $(\mathrm{P}<0,05)$. EEM: Error estándar de la media. Figura obtenida de Alfonso et al. (2011). Figure 2. Location during 24 h of lactating or pregnant rabbit females housed in standard cages vs. cages with platform, both provided with footrests. a, b, c Means for the gridded floor not sharing superscript differ significantly $(P<0.05)$. A, B Means for the footrest not sharing superscript differ significantly $(P<0.05)$. EEM: standard error of the mean. Figure obtained from Alfonso et al. (2011).

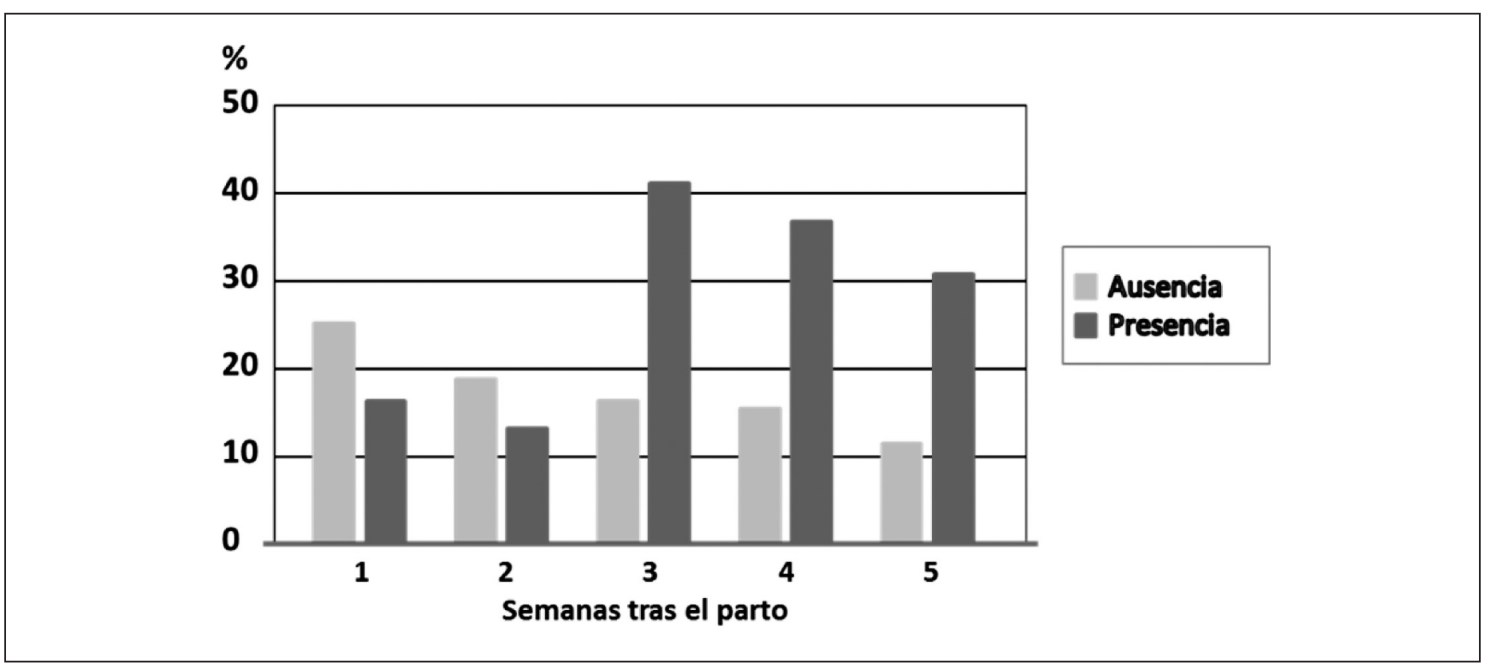

Figura 3. Porcentaje de tiempo que las conejas utilizan la plataforma durante la lactación en función de que sus gazapos estén presentes en la jaula (Presencia) o se coloquen en otra jaula y solo se les lleve a mamar una vez cada 24 horas (Ausencia). Figura obtenida de Mirabito (2003).

Figure 3. Percentage of time that rabbit females use the platform during lactation depending on whether their rabbits are present in the cage (Presencia) or placed in another cage and are only allowed to suckle once every 24 h (Ausencia). Figure obtained from Mirabito (2003). 
Los primeros experimentos con la plataforma ya indicaron que el problema más importante es la falta de higiene en la jaula por la caída de heces y orina sobre los animales situados debajo de la misma y por el acúmulo de heces sobre ella, así como la presencia de pododermatitis y de fracturas que no se producen en las jaulas estándar (Olivas et al., 2010). También más recientemente se han señalado éstos y otros problemas: peores condiciones higiénicas (Alfonso-Carrillo et al., 2014a; López et al., 2019), mayor frecuencia de episodios de agresividad frente al cuidador (López et al., 2019), aumento del tiempo de trabajo y muy elevada tasa de pododermatitis respecto a jaulas estándar (Lagardera et al., 2014).

Su efecto sobre los rendimientos productivos es, sin embargo, prácticamente nulo, pues se aproximan a los obtenidos en jaulas comerciales flat-deck en la mayoría de las experiencias. En algunos trabajos recientes se ha señalado un efecto positivo sobre el peso de la camada a las tres semanas de edad así como al destete (Barge et al., 2008), que se mantiene incluso hasta el final del periodo de cebo y está ligado a un mayor consumo y una tendencia a un menor índice de conversión (Alfonso-Carrillo et al., 2014b), pero en otros el efecto es justo el contrario (López et al., 2019). Índices importantes como fertilidad, prolificidad o mortalidad no han resultado diferentes a los de las jaulas convencionales prácticamente en ninguno de los experimentos de reproductoras en jaula con plataforma, pero lo encuentran Barge et al. (2008) en la mortalidad de la línea Bianca Italiana respecto a la línea Macchiata Italiana cuando ambas ocupan jaulas con plataforma, mostrando la primera una menor mortalidad durante toda la lactación $(19,9$ vs. 29,0\%) y, particularmente, al nacimiento y entre los 21-35 días postparto. Por el contrario, la línea Bianca Italiana reacciona negativamente en las jaulas estándar principalmente al nacimiento ( 12,0 vs. $7,2 \%$ de mortinatalidad). Los autores sugieren que podría haber líneas más apropiadas para la plataforma que producirían menos gazapos muertos al nacimiento, así como durante la lactación y al destete.

En definitiva, la plataforma se plantea como un elemento útil desde el punto de vista etológico y que no deteriora los resultados productivos -incluso puede mejorarlos como se ha indicado-, pero en el que deberían solucionarse los problemas de manipulación de los animales y de higiene para que su efecto beneficioso sobre el bienestar fuese real.

\section{Enriquecimiento de la jaula maternal mediante objetos}

Otros elementos enriquecedores del entorno de la jaula convencional de reproductoras, mejoradores del bienestar sin el impacto económico y estructural que podría tener el manejo en grupo o la implementación de jaulas con plataforma en las granjas comerciales, son los elementos para roer y el uso del reposapatas. Las conejas que disponen de reposapatas lo utilizan con alta frecuencia (entre el 58 y el $89 \%$ de su tiempo discurre sobre él), en cualquier estado fisiológico y edad (López et al., 2002), aunque a veces es en el periodo de lactación en el que destaca su uso (Alfonso et al., 2011) y otras es al final de la gestación (López et al., 2002). La utilización de reposapatas reduce el mal de patas en las reproductoras, según han mostrado de Jong et al. (2008) y Rosell y de la Fuente (2009), y su presencia en granja está ligada a un menor número de canales retiradas de la cadena de sacrificio (Fouchez et al., 2015), razones que lo aconsejan como una adecuada inversión. Sabemos, no obstante que, aunque el reposapatas pueda paliar el problema de pododermatitis, también puede dar lugar al acúmulo de suciedad en la jaula. El siguiente paso sería, por tanto, encontrar el modelo de reposapatas que se ajuste de manera pre- 
cisa al suelo de la jaula, o utilizar en las jaulas suelos completos de rejilla plástica que son los preferidos por los conejos (Princz et al., 2008, entre otros autores). Es importante, sin embargo, tener en cuenta que el tipo de suelo puede condicionar la limpieza de la jaula, indicando tanto veterinarios como cunicultores que ciertos slats plásticos son más difíciles de limpiar que los suelos de varilla metálica, con la correspondiente complicación por los problemas de higiene y sanitarios que comportan (Maertens, 2017), siendo así imprescindible continuar estudiando un adecuado diseño de la rejilla plástica. En relación con el suelo con cama de paja, éste ha sido cuestionado en los trabajos realizados al respecto, y rechazado en todos los experimentos en que los animales tenían posibilidad de elegir (hembras reproductoras: Ruis, 2006; Szendrö et al., 2013; gazapos en cebo: Gerencsér et al., 2014, entre otros autores); en contra de la opinión social casi generalizada, que considera que el suelo con paja es el idóneo para esta especie.

Respecto a los elementos destinados a roer o a entretenimiento de las conejas, han evolucionado desde una simple varilla de madera depositada en el suelo de la jaula, que por razones de higiene pasó a colgarse del techo o las paredes, hasta el estudio de "conglomerados" de madera enriquecidos con diversos productos de interés nutritivo como, por ejemplo, fibras específicas o micronutrientes tipo $\mathrm{Ca}, \mathrm{P}, \ldots$. Estos productos tienen interés por sus dos vertientes, comportamental y productiva. Al respecto, hace años que los nutrólogos advertían sobre la importancia de ofrecer un buen pienso, ajustado a las necesidades del animal, antes de dar suplementos vitamínico-minerales que pudieran desequilibrar la dieta. Parece, sin embargo, que en las jaulas con taco enriquecido las hembras y los gazapos presentan pesos superiores a las que disponen solo del taco de pasta de madera sin enriquecer, además de aumentar el comportamiento locomotor y el de ingesta (Maertens et al., 2012), aunque en el experimento de Bignon et al. (2012) ni el comportamiento, ni la producción lechera, ni el peso de la camada se modifican. Evidentemente, tanto el efecto del taco enriquecido sobre el equilibrio o desequilibrio de los otros nutrientes, como el coste de la incorporación de estos elementos enriquecedores deberían ser contrastados, pues no lo están a día de hoy. Incluso sin adición especial de componentes nutritivos, las conejas prefieren y aumentan el patrón de comportamientos con el taco de conglomerado respecto a únicamente la varilla de madera, siendo la paja el material de preferencia entre los mencionados, según resultados de Rommers et al. (2014b). La paja es un producto hacia el que las conejas se sienten atraídas inmediatamente después de su colocación en la jaula, pero sobre el que van reduciendo progresivamente su interés a lo largo de la primera hora de contacto con el mismo (López et al., 2004), lo que respondería a un comportamiento exploratorio característico de los animales frente a la novedad (Wood-Gush y Vestergaard, 1991).

\section{Otros aspectos de bienestar con influencia sobre los resultados zootécnicos}

La duración del ciclo reproductivo se ha sugerido como factor de bienestar a considerar, especialmente comparando el popular ciclo de 42 días (cubrición día 11 post-parto) con los otros más extensivos de 49 o 56 días (cubrición día 18 o día 25 post-parto, respectivamente). Gerencsér et al. (2011) encuentran que el ciclo de 56 días mejora la fertilidad, el peso de la coneja al parto y su condición corporal en la semana $4^{a}-5^{a}$ post-parto. También mejora la supervivencia de las reproductoras, de modo que este ritmo puede ser ventajoso desde el punto de vista de bienes- 
tar de la coneja, según estos autores. Por el contrario, Alfonso-Carrillo et al. (2014b) indican que ciclos largos no favorecen a la coneja pero sí a la camada, la cual presenta mejor peso e índice de transformación y menor mortalidad al final del periodo de cebo. Por otra parte, algunos autores señalan que la menor productividad numérica de los ciclos largos ( $n^{\circ}$ de gazapos/hembra/año) puede tener un alto impacto económico y condicionar su aplicación (Gerencsér et al., 2011). Otros autores cuestionan el beneficio de prolongar la lactación más allá de las 4 semanas porque "no es conforme a la biología reproductiva de las conejas" (Schlolaut et al., 2013). Además, el mayor contacto de madre y gazapos, junto al consumo del pienso materno, puede aumentar el riesgo de infecciones en los gazapos y de mamitis en las conejas, así como retrasar el desarrollo digestivo de los jóvenes, con el correspondiente riesgo de patologías entéricas (Schlolaut et al., 2013). En consecuencia, parece que sobre la duración del ciclo y el manejo del mismo todavía debemos seguir experimentando.

En relación con el bienestar de las conejas también hay que señalar el papel que pueden jugar las feromonas, aunque solamente hemos localizado un experimento al respecto y sería necesario contrastar los resultados. Así, Bouvier y Jacquinet (2008), elaboran al efecto una feromona a base de una mezcla de ácidos grasos, ésteres y dímeros de ácidos grasos secretados en la piel del vientre de las conejas durante los 2-3 días previos al parto. Pulverizada en la sala de maternidad en diferentes granjas comerciales 2 o 3 días antes del parto, provoca tranquilidad en las conejas y facilidad en su manipulación, según indicaron los propios cunicultores, mejorándose la fertilidad, el tamaño de la camada y la viabilidad al parto.

Finalmente, hay que señalar que otras prácticas de manejo pensadas o desarrolladas con otros objetivos también pueden contri- buir a mejorar el bienestar de las conejas. Así, la aplicación de la hormona GnRH vía vaginal en conjunción con el semen es una importante mejora en el manejo reproductivo de las conejas pues disminuye el estrés de las mismas, habiendo sido calificada por expertos europeos como un "interesante aporte para el bienestar de las conejas" (de Jong et al., 2011). La respuesta fisiológica y productiva de diferentes análogos de GnRH y sus dosis óptimas para la vía vaginal siguen investigándose (Munari et al., 2019). No obstante, este manejo, además de moderar el estrés, reduce la mano de obra, mejora las condiciones de trabajo del ganadero, aminora el riesgo sanitario y disminuye el coste al no necesitar jeringuillas ni agujas (Martín, 2016), de modo que se está utilizando en muchas de las granjas comerciales que aplican inseminación artificial.

\section{Comentarios finales}

La peculiar situación de la especie cunícola como animal de renta, de compañía y de laboratorio y su simultánea presencia como silvestre, unido al interés del consumidor occidental por el acercamiento a la naturaleza en general y a los comportamientos "naturales" propios de las especies (etología cunícola en nuestro caso) en aras del supuesto bienestar de las mismas, hace que se estudien las posibilidades del manejo en grupos de las reproductoras domésticas y que repetidamente se compruebe su dificultad. Esta situación sugiere que tal vez una condición para la constitución de un grupo de conejas sea, precisamente, el desarrollo de la agresividad y de la sumisión necesarias para establecer una jerarquía que permita la estabilidad y su supervivencia como grupo. Y posiblemente ocurre lo mismo con los machos, aunque en este sexo las expresiones agresivas son más radicales despertando, tal vez por ello, escaso in- 
terés que los machos vivan en grupo o incluso la mejora de su bienestar, aspecto prácticamente inexistente en la literatura científica relativa a producción de carne.

Ante la incapacidad de controlar o gestionar tanto la agresividad entre hembras como la constitución de jerarquías en las granjas, parece que el interés de los científicos por el posible enriquecimiento comportamental de las conejas criadas en grupo ha disminuido, ya que en realidad se enriquece levemente, como hemos indicado. Por otra parte, ha creado alarma la probable reducción del bienestar que comporta, sea del grupo o sea de algunos miembros del mismo, lo cual, a su vez, se traduce en un deterioro de los resultados zootécnicos, incluida la mortalidad y morbilidad de adultos y de jóvenes. Encauzar esa agresividad es imprescindible si se quieren criar conejos en base a grupos de reproductoras.

No obstante, esta respuesta biológica probablemente no es fácil de gobernar y de momento se desconoce el procedimiento. Por ello, a nivel práctico y considerando las diferencias en los sistemas de producción de los países productores, no parece razonable que se establezca legislación común en la UE sobre este aspecto del bienestar cunícola. Y en los países, europeos o no, que legislen al respecto deberían tener muy en consideración esta circunstancia señalada en todos los experimentos realizados al efecto. Al respecto, y refiriéndose a conejos de laboratorio, DiVincenti y Rehrig (2016) indican: "Por lo tanto, las partes interesadas deben considerar qué especies de laboratorio podrían beneficiarse del alojamiento social en cautividad antes de promulgar cambios generalizados, que en realidad podrían ser contraproducentes para mejorar el bienestar de los animales de laboratorio". Esta reflexión es perfectamente aplicable para los conejos de producción de carne.

Favorecer el contacto visual, olfativo, auditivo, utilizar feromonas $u$ otras sustancias o manejos durante el periodo de estancia en la jaula individual, hacer un diseño ad hoc de la jaula o del parque, o incluso el propio proceso de selección genética, podrían tal vez ser útiles para atemperar esta respuesta biológica propia de la especie y que se mantiene claramente en los conejos domésticos. Refinar el enriquecimiento de la jaula individual de las conejas y de los machos a base de la mejora del rendimiento de la superficie útil o de la inclusión de elementos enriquecedores apropiados parece el camino a recorrer hasta entonces, tanto desde el punto de vista experimental como aplicado.

\section{Referencias bibliográficas}

Alfonso C, Martín E, Esteban M, De Blas C, GarcíaRebollar P, García-Ruiz Al (2011). Efecto del tipo de jaula sobre el comportamiento de conejas reproductoras en distintos estados fisiológicos. Actas del XXXVI Symposium de Cunicultura de ASESCU, 12-13 de mayo, Peñíscola, España. http://oa.upm.es/12049

Alfonso-Carrillo C, Martín E, De Blas C, Ibáñez MA, García-Rebollar P, García-Ruiz Al (2014a). Effect of cage type on the behaviour pattern of rabbit does at different physiological stages. World Rabbit Science 22: 59-69. https://doi.org/ 10.4995/wrs.2014.1396

Alfonso-Carrillo C, García-Rebollar P, De Blas C, Ibáñez MA, García-Ruiz Al (2014b). Effect of late weaning and use of alternative cages on performance of does, suckling and fattening rabbits under extensive reproductive management. Livestock Science 167: 425-434. https:// doi.org/10.1016/j.livsci.2014.05.018

Andrist CA, Bigler LM, Würbel H, Roth BA (2012). Effects of group stability on aggression, stress and injuries in breeding rabbits. Applied Animal Behaviour Science 142: 182-188. https:// doi.org/10.1016/j.applanim.2012.10.017

Andrist CA, Bigler L, Würbel H, Roth BA (2014). Masking odour when regrouping rabbit does: 
Effect on aggression, stress and lesions. Livestock Science 170: 150-157. https://doi.org/10. 1016/j.livsci.2014.10.017

Barge P, Masoero G, Chicco R (2008). Raising rabbit does in platform cages. Proceedings of the 9th World Rabbit Congress, 10-13 de junio, Verona, Italia, pp. 1153-1157.

Bignon L, Travel A, Galliot P, Souchet C, Davoust C, Weissman $D$ (2012). Gnawing blocks in rabbit cages: impact on the behaviour and performance of does and fattening rabbits. Proceedings of the 10th World Rabbit Congress, 3-6 de septiembre, Sharm El-Sheikh, Egipto, pp. 1051-1055.

Bouvier AC, Jacquinet C (2008). Pheromone in rabbits: preliminary technical results on farm use in France. Proceedings of the 9th World Rabbit Congress, 10-13 de junio, Verona, Italia, pp. 303-307.

Buijs S, Maertens L, Hermans K, Vangeyte J, Tuyttens FAM (2015). Behaviour, wounds, weight loss and adrenal weight of rabbit does as affected by semi-group housing. Applied Animal Behaviour Science 172: 44-51. https://doi.org/ 10.1016/j.applanim.2015.09.003

BVAAWF / FRAME / RSPCA / UFAW (1993). Refinements in rabbit husbandry. Laboratory Animals 27: 301-329.

Carneiro M, Rubin CJ, Di Palma F, Albert FW, Alföldi J, Barrio AM, Pielberg G, Rafati N, Sayyab S, Turner-Maier J, Younis S, Afonso S, Aken B, Alves JM, Barrell D, Bolet G, Boucher S, Burbano HA, Campos R, Chang JL, Duranthon V, Fontanesi L, Garreau H, Heiman D, Johnson J, Mage RG, Peng Z, Queney G, Rogel-Gaillard C, Ruffier $M$, Searle $S$, Villafuerte $R$, Xiong $A$, Young $S$, Forsberg-Nilsson K, Good JM, Lander ES, Ferrand N, Lindblad-Toh K, Andersson L (2014). Rabbit genome analysis reveals a polygenic basis for phenotypic change during domestication. Science 345(6200): 1074-1079. https://doi. org/10.1126/science.1253714

Cervera C, Martínez-Paredes E, Machado L, Villagrá A (2016). Efecto sobre hembras y gazapos de la cría de conejas reproductoras en jaulas colectivas. Actas del XLI Symposium de Cunicultura de ASESCU, 12 y 13 de mayo, Hondarribia, España, pp. 130-133.
Cervera C, Martínez-Paredes E, Machado L, Villagrá A (2017). Producción de conejas en sistemas de alojamiento individual o colectivo en semigrupo. Actas del XLII Symposium de Cunicultura de ASESCU, 11 y 12 de mayo, Murcia, España, pp.107-110.

Cervera C, Gómez EA, Pérez Fuentes S, Villagrá A (2018). Bienestar y salud en conejas reproductoras. (Proyecto INIA CUN2014-00001-00-00). Actas del 43 Symposium de Cunicultura de ASESCU, 30 y 31 de mayo, Calamocha, España, 33-42.

de Jong IC, Reimert H, Rommers JM (2008). Effect of floor type on footpad injuries in does: a pilot study. Proceedings of the 9th World Rabbit Congress, 10-13 de junio, Verona, Italia, pp. 1171-1175.

de Jong IC, Reuvekamp BFJ, Rommers JM (2011). A welfare assessment protocol for commercially housed rabbits. Wageningen UR Livestock Research, Report 532, 24 pp.

DiVincenti L, Rehrig AN (2016). The Social Nature of European Rabbits (Oryctolagus cuniculus). Journal of the American Association for Laboratory Animal Science 55(6): 729-736.

Fouchez L, Tessereau A, Greffard B, Bruhier A (2015). Influence des pratiques d'élevage sur le taux de saisie des lots de lapins en abattoir. 16èmes Journées de la Recherche Cunicole, 2425 de noviembre, Le Mans, France, pp. 189-192.

Gerencsér Zs, Matics Zs, Nagy I, Szendrö Zs (2011). Effect of light colour and reproductive rhythm on rabbit doe performance. World Rabbit Science 19: 161-170. https://doi.org/10.4995/ wrs.2011.827

Gerencsér Zs, Szendrö K, Szendrö Zs, Odermatt M, Radnai I, Nagy I, Dal Bosco A, Matics Zs (2014) Effect of floor type on behavior and productive performance of growing rabbits. Livestock Science 165: 114-119. https://doi.org/10.1016/ j.livsci.2014.04.022

González-Redondo P, González-Mariscal G, López M, Fernández-Carmona J, Finzi A, Villagrá $A$ (2015). Comportamiento materno y bienestar de la coneja doméstica y silvestre y su camada. ITEA-Información Técnica Económica Agraria 111(4): 326-347. https://doi.org/10.12706/itea. 2015.021 
Graf S, Bigler L, Failing K, Würbel H, Buchwalder $T$ (2011). Regrouping rabbit does in a familiar or novel pen: Effects on agonistic behaviour, injuries and core body temperature. Applied Animal Behaviour Science 135: 121-127. https:// doi.org/10.1016/j.applanim.2011.10.009.

Hoy St, Schuh D (2004). Sociometric investigations in groups of wild and domestic rabbits with one buck and two or three does. Proceedings of the 8th World Rabbit Congress, 7-10 de septiembre, Puebla, México, pp.1235-1240.

Hoy St, Verga M (2006). Welfare indicators. En: Recent Advances in Rabbit Sciences (Ed. L. Maertens y P. Coudert) pp. 71-74. ILVO, Melle. Belgium.

Lagardera G, Solaz S, Martínez-Paredes E, Cervera C (2014). Comparación de algunos aspectos del manejo y de la sanidad de conejas lactantes según el diseño de la jaula de maternidad. Actas del XXXIX Symposium de Cunicultura de ASESCU, 29-30 de marzo, Tudela, España, pp. 42-45.

López M, María G, Paniagua P (2002). The effect of cage size on the behavior of the reproductive doe. COST Action 848. 2nd Meeting of Workgroup 2, 15-16 de marzo, Stuttgart, Hohenheim, $10 \mathrm{pp}$.

López M, Carrilho MC, Gómez C (2004). Evaluation of the use of straw as an entertainment in $\mathrm{Gi}$ gante de España rabbit cages: The effect of the placing of the straw in cage on the behaviour. Proceedings of the 8th World Rabbit Congress, 7-10 de septiembre, Puebla, México, pp. 12411246.

López M, Gómez EA, Cervera C (2019). Comparación de jaulas de maternidad con plataforma frente a jaulas convencionales en una prueba de campo. Actas del 44 Symposium de Cunicultura de ASESCU, 5-6 de junio, Aranda de Duero, España, pp. 27-32.

Machado LC, Martínez-Paredes E, Paragliota F, Cervera C (2016). Performance and health status of primiparous rabbit does housed in individual and collective cages. Proceedings of the 11th World Rabbit Congress, 15-18 de junio, Qingdao, China, pp. 695-698.

Maertens L, Buijs S, Davoust C (2012). Gnawing blocks as cage enrichment and dietary supple- ment for does: performance, intake and behavior. Proceedings of the 10th World Rabbit Congress, 3-4 de septiembre, Sharm El-Sheikh, Egipto, pp. 1039-1043.

Maertens L, Buijs S, Tuyttens FAM (2015). L'impact d'un logement temporairement en groupe et du sol sur le bien-être des lapines. 16èmes Journées de la Recherche Cunicole, 24-25 de noviembre, Le Mans, France, pp. 215-218.

Maertens L, Buijs S (2016). Production performances of rabbit does in a part-time group housing system. Proceedings of the 11th World Rabbit Congress, 15-18 de junio, Qingdao, China, pp. 711-714.

Maertens L (2017). Farmers experiences with park housing of rabbits. 20th International Symposium on housing and diseases of rabbits, furproviding animals and pet animals, 17-18 de mayo, Celle, Alemania, pp. 37-41.

Maertens L, De Bie Y (2017). Logement de lapines "part-time" en groupe: résultats dans un élevage équipé avec des parcs polyvalents. 17èmes Journées de la Recherche Cunicole, 21-22 de noviembre, Le Mans, France, pp. 55-58.

Martín M (2016). Inseminación artificial, un nuevo método en cunicultura. Cunicultura.info 30/ 08/2016. Disponible en https://cunicultura.info/ inseminacion-artificial-nuevo-metodo-cunicultura/ (Consultado: 21 octubre 2019).

Martínez-Paredes E, Ródenas L, Cervera C (2019). Estación, tamaño y densidad del grupo de conejas durante la recría y productividad. Actas del 44 Symposium de Cunicultura de ASESCU, 56 de junio, Aranda de Duero, España, pp. 22-26.

Matics Zs, Szendrö Zs, Radnai I, Farkas TP, Kasza R, Kacsala L, Nagy I, Szabó RT, Terhes K, Gerencsér Zs (2017). ANIHWA - Experimental results at Kaposvár University. 20th International Symposium on housing and diseases of rabbits, furproviding animals and pet animals. 17-18 de mayo, Celle, Alemania, pp. 27-36.

Mikó A, Matics Zs, Gerencsér Zs, Radnai I, Odermatt M, Nagy I, Szendrö Zs (2012). Location preference of lactating rabbit does and their kits in pens with elevated platform. Proceedings of the 
10th World Rabbit Congress, 3-6 de septiembre, Sharm El-Sheikh, Egipto, pp. 1029-1032.

Mirabito L, Galliot P, Souchet C (2000). Effect of different ways of cage enrichment on the productive traits and mortality of fattening rabbits. Proceedings of the 7th World Rabbit Congress, 4-7 de julio, Valencia, España. Vol B, pp. 447-451.

Mirabito L (2003). Logement et bien-être du lapin : les nouveaux enjeux. 10èmes Journées de la Recherche Cunicole, INRA-ITAVI, 19-20 de noviembre, Paris, France, pp. 163-172.

Mirabito L, Boquier C (2005). Développement d'un système $d$ 'alimentation séparée des mères et des jeunes avant sevrage. 11èmes Journées de la Recherche Cunicole, 29-30 de noviembre, Paris, France, pp. 49-52.

Mirabito L, Galliot P, Souchet C, Dumont F, Thomeret $F$ (2005a). Logement collectif des lapines repro-ductrices : Conséquences zootechniques. 11 èmes Journées de la Recherche Cunicole, Paris, 29-30 de noviembre, Paris, France, pp. 53-56.

Mirabito L, Dumont F, Galliot P, Souchet C (2005b). Logement collectif des lapines reproductrices Conséquences sur le comportement. 11èmes Journées de la Recherche Cunicole, 29-30 de noviembre, Paris, France, pp. 57-60.

Mirabito L, Galliot P, Souchet C (2005c). Effet de la surface disponible et de l'aménagement des cages sur les perfor-mances zootechniques et le comportement des lapines. 11 èmes Journées de la Recherche Cunicole, 29-30 de noviembre, Paris, France, pp. 61-64.

Munari C, Ponzio P, Alkhawagah AR, Schiavone A, Mugnai C (2019). Effects of an intravaginal $\mathrm{GnRH}$ analogue administration on rabbit reproductive parameters and welfare. Theriogenology 125: 122-128. https://doi.org/10.1016/ j.theriogenology.2018.10.024

Mullan S, Saunders R (2019). European Rabbits (Oryctolagus cuniculus). En: Companion Animal Care and Welfare: The UFAW Companion Animal Handbook. (Ed. James Yeates) pp. 163184. Ufaw Animal Welfare Series; Wiley-Blackwell, Hoboken. Nueva Jersey, EEUU. https:// doi.org/10.1002/9781119333708.ch7
Negretti P, Bianconi G, Finzi A (2010). Postura y comportamiento del conejo para determinar la altura de la jaula en relación con el bienestar animal. Actas del XXXV Symposium de Cunicultura, ASESCU, 27-28 de mayo, Segovia, España, pp. 54-58.

Olivas I, Gómez E, Laínez M, Villagrá A (2010). Jaulas con plataforma ¿mejorando el bienestar animal? Un estudio piloto. Boletín de Cunicultura 162: 27-40.

Paillard G (1970). L'élevage en groupe et l'organisation de la production et des marchés du lapin de chair. Coloque national du lapin, Paris. Cit. en Cuniculture, 132, 23 (6), 262, 1996.

Princz Z, Nagy I, Radnai I, Gerencsér Zs, Szendrö Zs (2008). Effect of the floor type and stocking density on the productive performance of growing rabbits. Proceedings of the 9th World Rabbit Congress, 10-13 de junio, Verona, Italia, pp. 1225-1228.

Ródenas L, Martínez-Paredes E, Cervera C (2019). Estrés y vida productiva en conejas. Actas del 44 Symposium de Cunicultura de ASESCU, 5-6 de junio, Aranda de Duero, España, pp. 17-21.

Rommers JM, Boiti C, de Jong IC, Brecchia G (2006). Performance and behaviour of rabbit does in a group-housing system with natural mating or artificial insemination. Reproduction Nutrition Development 46: 677-687. https:// doi.org/10.1051/rnd:2006038

Rommers JM, Kemp B, Houwers HW, Gunnink H, de Jong IC (2012). Description of nestbox visits and suckling events in a group housing system for rabbit does as compared to individual cages. World Rabbit Science 20: 231-240. https:// doi.org/10.4995/wrs.2012.1231

Rommers JM, Reuvekamp BJF, Gunnink $\mathrm{H}$, de Jong IC (2014a). Effect of hiding places, straw and territory on aggression ingroup-housed rabbit does. Applied Animal Behaviour Science 157: 117-126. https://doi.org/10.1016/j.applanim. 2014.05.011

Rommers JM, Bracke MBM, Reuvekamp B, Gunnink H, de Jong IC (2014b). Cage enrichment: rabbit does prefer straw or a compressed wooden block. World Rabbit Science 22: 301-309. https://doi.org/10.4995/wrs.2014.1353 
Rommers J, de Greef K (2017). Towards part-time group-housing of lactating rabbit does? 20th International Symposium on housing and diseases of rabbits, furproviding animals and pet animals. 17-18 de mayo, Celle, Alemania, pp. 3-13.

Rosell JM y de la Fuente LF (2009). Effect of footrests on the incidence of ulcerative pododermatitis in domestic rabbit does. Animal Welfare 18: 199-204.

RWAF (2019). Aggression in Rabbits. Disponible en https://rabbitwelfare.co.uk/rabbit-care-advice/aggression-rabbits/ (Consultado: 1 junio 2019).

Ruis M, Coenen E (2004). A group-housing system for rabbit does in commercial production: a new approach. Proceedings of the 8th World Rabbit Congress, 7-10 de septiembre, Puebla, México pp. 1501-1506.

Ruis M, Rommers J, van del Gaag M (2005). Reproductive performance and behaviour of does in group-housing. Joint Scientific Meeting WG1 and WG2. 23-25 de junio, Palermo, Italia.

Ruis M (2006). Group housing of breeding does. In: Recent advances in rabbit sciences (Ed. L. Maertens and P. Coudert) pp. 99-105. ILVO, MeIle, Belgium.

Schlolaut W, Hudson R, Rödel HG (2013). Impact of rearing management on health in domestic rabbits: A Review. World Rabbit Science 21: 145-159. https://doi.org/10.4995/wrs.2013.1029

Scottish Government (2018). Pet Rabbit Welfare Guidance. The Scottish Government, OGL, Edinburgh. Disponible en www.gov.scot (Consultado: 1 junio 2019).

Szendrö Zs (2009). Environmental conditions required for the rabbits' wellbeing. 21st Hungarian Conference on Rabbit Production, 27 de mayo, Kaposvár, Hungary. Abstract: World Rabbit Science 2010, 18(1): 37.
Szendrö Zs, Dezséry B, Odermatt M, Gerencsér Zs, Nagy I, Molnár Sz, Mikó A, Radnai I, Matics Zs (2010). Comparison of reproductive performance of rabbit does housed individually or in groups. 22nd Hungarian Conference on Rabbit Production, Kaposvár, Hungary, 26 de mayo. Abstract: World Rabbit Science 2011, 19(2): 114.

Szendrö Zs, McNitt JI (2012). Housing of rabbit does: Group and individual systems: A review. Livestock Science 150: 1-10. https://doi.org/ 10.1016/j.livsci.2012.09.017

Szendrö Zs, Mikó A, Odermatt M, Gerencsér Zs, Radnai I, Dezséry B, Garai E, Nagy I, Szendrö K, Matics Zs (2013). Comparison of performance and welfare of single-caged and group-housed rabbit does. Animal 7(3): 463-468. https://doi. org/10.1017/S1751731112001760

Szendrö Zs, McNitt Jl, Matics Zs, Mikó A, Gerencsér Zs (2016). Alternative and enriched housing systems for breeding does: a review. World Rabbit Science 24 (1): 1-14. https://doi.org/10. 4995/wrs.2016.3801

Villalba T (2019). Código de Protección y Bienestar Animal. Agencia Estatal Boletín Oficial del Estado. Disponible en https://www.boe.es/legislacion/codigos/ (Consultado: 21 octubre 2019).

Von Holst D, Hutzelmeyer $H$, Kaetzke $P$, Khaschei M., Schönheiter R (1999). Social rank, stress, fitness and life expectancy in wild rabbits. Naturwissenschaften 86: 388-393. https://doi. org/10.1007/s001140050638.

Wood-Gush DGM, Vestergaard K (1991). The seeking of novelty and its relation to play. Animal Behaviour 42: 599-606. https://doi.org/10.1016/ S0003-3472(05)80243-X

(Aceptado para publicación el 24 de enero de 2020) 